\title{
Assessing field performance of ultrasensitive rapid diagnostic tests for malaria: a systematic review and meta-analysis
}

\author{
Celestin Danwang ${ }^{1,2^{*}}$, Fati Kirakoya-Samadoulougou ${ }^{3}$ and Sekou Samadoulougou 4,5
}

\begin{abstract}
Background: To overcome the limitations of conventional malaria rapid diagnostic tests (CRDTs) in diagnosing malaria in patients with low parasitaemia, ultrasensitive malaria rapid diagnostic tests (URDTs) have recently been developed, with promising results under laboratory conditions. The current study is the first meta-analysis comparing the overall sensitivity, and specificity of newly developed ultrasensitive Plasmodium falciparum malaria RDT (Alere ${ }^{\mathrm{TM}}$ Ultra-sensitive Malaria Ag P. falciparum RDT) with the CRDT conducted in the same field conditions.

Methods: PubMed, EMBASE, Cochrane infectious diseases group specialized register, and African Journals Online (AJOL) were searched up to $20^{\text {th }}$ April 2021. Studies with enough data to compute sensitivity and specificity of URDT and CRDT were retrieved. A random-effect model for meta-analysis was used to obtain the pooled sensitivity and specificity.
\end{abstract}

Results: Overall, 15 data sets from 14 studies were included in the meta-analysis. The overall sensitivity of the Alere ${ }^{\mathrm{TM}}$ ultra-sensitive Malaria Ag P. falciparum RDT regardless of the reference test and the clinical presentation of participants, was 55.5\% (95\% confidence interval [Cl]: 45.5; 65.0), while the sensitivity regardless of the reference test and the clinical presentation of participants, was $42.9 \%(95 \% \mathrm{Cl}: 31.5 ; 55.2)$ for the cRDT performed in the same field conditions. When PCR was used as reference test, the sensitivity of URDT was $60.4 \%$ (95\% Cl: 50.8; 69.2), while the sensitivity was $49.4 \%$ ( $95 \% \mathrm{Cl}: 38.2 ; 60.6)$ for the CRDT. The pooled specificity of URDT regardless of the reference test and the clinical presentation of participants was $98.6 \%$ (95\% Cl: $97.1 ; 99.4)$, and the pooled specificity of CRDT regardless of the reference test and the clinical presentation of participants was $99.3 \%$ (95\% Cl: 98.1 ; 99.7). When PCR was used as reference test the specificity of uRDT and CRDT was $97.5 \%$ (95\% Cl: 94.1 ; 98.9) and 98.2\% (95\% Cl: $95.5 ;$ 99.3). Regardless of the reference test used, the sensitivity of Alere ${ }^{\mathrm{TM}}$ Ultra-sensitive Malaria Ag P. falciparum RDT in symptomatic patients was $72.1 \%$ (95\%Cl: $67.4 ; 76.4)$, while sensitivity of CRDT was $67.4 \%$ (95\%Cl: $57.6 ; 75.9)$.

Conclusion: Findings of the meta-analysis show that Alere ${ }^{\mathrm{TM}}$ Ultra-sensitive Malaria Ag P. falciparum RDT compared to cRDT performed in the same field conditions has higher sensitivity but lower specificity although the difference is not statistically significant.

*Correspondence: danram07@yahoo.fr

${ }^{1}$ Department of Surgery and Specialties, Faculty of Medicine and Biomedical Sciences, University of Yaoundé I, Yaoundé, Cameroon

Full list of author information is available at the end of the article

\section{Background}

Even though the global burden of malaria has been reduced since 2000 , in 2018 , nearly 228 million new cases of malaria were recorded globally, and there were close to 405.000 excess death caused by malaria [1]. Among those deaths, children [2] and pregnant [3] women represent the most vulnerable population. To reduce and 
eliminate malaria infections, the World Health Organization (WHO) recommends the use of the « Test, Treat and Track» strategy [4]. The aim of this strategy is to make sure that every suspected case is tested using a confirmation test, and every confirmed case is treated with the appropriate anti-malaria medication [4].

In people living in areas where malaria is prevalent, and in pregnant women, malaria diagnosis can be challenging. In the former, because every suspected case must be diagnosed and treated, even those with parasitaemia below the detection threshold of conventional rapid diagnostic tests (cRDTs) for malaria, and in the latter, because of the ability of Plasmodium falciparum to bind to the placenta, which can lead to parasite densities in peripheral blood below the detection threshold of the most used cRDTs and light microscopy, hence the need for ultrasensitive diagnostic tests (uRDTs) [5].

Currently, nucleic acid amplification tests are known to be sensitive to detect these low-density infections $[6,7]$. Nonetheless, these methods are limited to well-equipped laboratory settings due to their inherent complexity and need for sophisticated laboratory facilities. Recently, to fulfil the demand for diagnostic tests that are cheaper, faster, with high-sensitivity and deployable in the field, uRDT was developed [8]. Like cRDTs, which detect proteins such as histidine-rich protein 2 (HRP2), aldolase, and parasite lactate dehydrogenase (pLDH), they are based on the immunodetection of HRP2 and exhibit promising results when their performance is assessed in laboratory conditions [8].

However, no study has hitherto evaluated through meta-analysis the performance of uRDT under field conditions. This first systematic review with meta-analysis aimed to compare the overall sensitivity, and specificity of newly developed ultrasensitive malaria RDT (Alere ${ }^{\mathrm{TM}}$ Ultra-sensitive Malaria Ag P. falciparum RDT) with the cRDT conducted in the same field conditions.

\section{Methods}

The review is conducted in accordance with the recommendations for diagnostic test accuracy meta-analysis in the Cochrane Handbook for Systematic Reviews [9] and is reported with respect to the Preferred Reporting Items for Systematic Review and Meta-analysis of Diagnostic Test Accuracy Studies [10]. The current review is registration with PROSPERO CRD42021227784.

\section{Search strategy}

PubMed, EMBASE, Cochrane infectious diseases group specialized register, and African Journals Online (AJOL) were searched from inception up to 20 April 2021 with the following terms: ("malaria[tiab]" OR "malaria [MESH]") AND ("ultrasensitive"[tiab] OR "highly sensitive" [tiab] OR "hypersensitive"[tiab] OR "highsensitive"[tiab] OR "high sensitive"[tiab] OR "RDT"[tiab]). The search strategy used in PubMed which was adapted to fit with other databases is presented in Additional file 1: Table S1.

After bibliographic search, the titles and abstracts were screened for eligibility and duplicates were removed. Full texts of potentially eligible articles were retrieved and assessed for final inclusion independently by two reviewers, with discrepancy between both resolved by discussion.

\section{Eligibility criteria}

Both observational and experimental studies reporting enough data to compute sensitivity and specificity of uRDT and cRDT in the same setting and comparing both with the same reference test were included in the metaanalysis. Only studies conducted on the field (not in the laboratory), regardless of the language and year of publication were retained. We excluded editorials, reviews, letters, commentaries, and studies lacking key data.

\section{Quality assessment}

The Quality Assessment of Diagnostic Accuracy Studies 2 (QUADAS-2) was used independently by two reviewers to assess the quality of included studies [11]. Disagreement between the two reviewers were resolved by discussion.

\section{Data extraction}

The following information was retrieved on a preconceived data extraction form by one reviewer: the name of the first author, the country where the study was conducted, the year of publication, the characteristics of the study population in terms of symptoms and age, the presence of pregnant women in the sample, the commercial name of the uRDT and reference test used in the study, the storage condition of the uRDT, the number of true positive (TP), true negative (TN), false positive (TP), false negative (FN).

Articles reporting on diagnostic performance of uRDT in different age categories (e.g., adults and children) or in which uRDT was compared with more than one reference test were divided in separated data sets. Thereafter, a second reviewer checked the concordance between data extracted and the content of the article prior to the data synthesis and analysis.

\section{Statistical analysis}

The "meta" package within $\mathrm{R}$ software version 4.0.2 was used for analysis [12,13]. A random-effect model was used to obtain the overall summary effect of studies reporting enough data to compute the sensitivity, and 
specificity. The Clopper-Pearson method was used to compute the confidence intervals and the maximum-likelihood estimator was used to estimate the between-study variance. The QUADAS-2 score was used to estimate the risk of bias in included studies. A P-value of 0.05 was considered statistically significant in all the analysis.

\section{Results}

\section{Search results}

The bibliographic search yielded 1440 articles. The screening based on title and abstracts and full text allowed to retain 15 data sets from 14 studies for the quantitative synthesis as depicted in the Prisma flow diagram (Fig. 1).

\section{Characteristics of the included studies}

Studies were published between 2018 and 2021, with 9 out of 14 conducted in sub-Saharan African countries. Two out of the 14 studies were from Myanmar [14, 15], two from Tanzania [16, 17], while Benin [18], Cambodia [19], Ethiopia [20], Indonesia [21], Kenya [22], Mozambique [23], Papua New Guinea [24], Ghana [25],
Colombia [26], and The Gambia [27] were represented by one study (Table 1).

Eight studies (57.1\%) were conducted in patients regardless of the presence of symptoms, while two (14.3\%) were conducted in symptomatic patients and four $(28.6 \%)$ in asymptomatic patients. The mean/median age of the study population ranged from 4 to 36 years as reported by seven studies. Four (28.6\%) studies were conducted in pregnant women (Table 1). Plasmodium falciparum was the species targeted by the uRDT test in all the studies, and all the uRDT were from the same manufacturer (abbott Alere ${ }^{\mathrm{TM}}$ Ultra-sensitive Malaria Ag P. falciparum RDT). The reference test was mainly PCR (10 data sets), and ultrasensitive PCR (4 data sets) (Table 1). The risk of bias in studies included in the review ranged from low to moderate and is summarized in Fig. 2.

\section{Meta-analysis of sensitivity and specificity \\ Sensitivity}

Even if the difference was not statistically significant, the Alere $^{\mathrm{TM}}$ Ultra-sensitive Malaria Ag $P$. falciparum RDT had a higher sensitivity than the cRDT performed in the same field conditions. The overall sensitivity of the uRDT

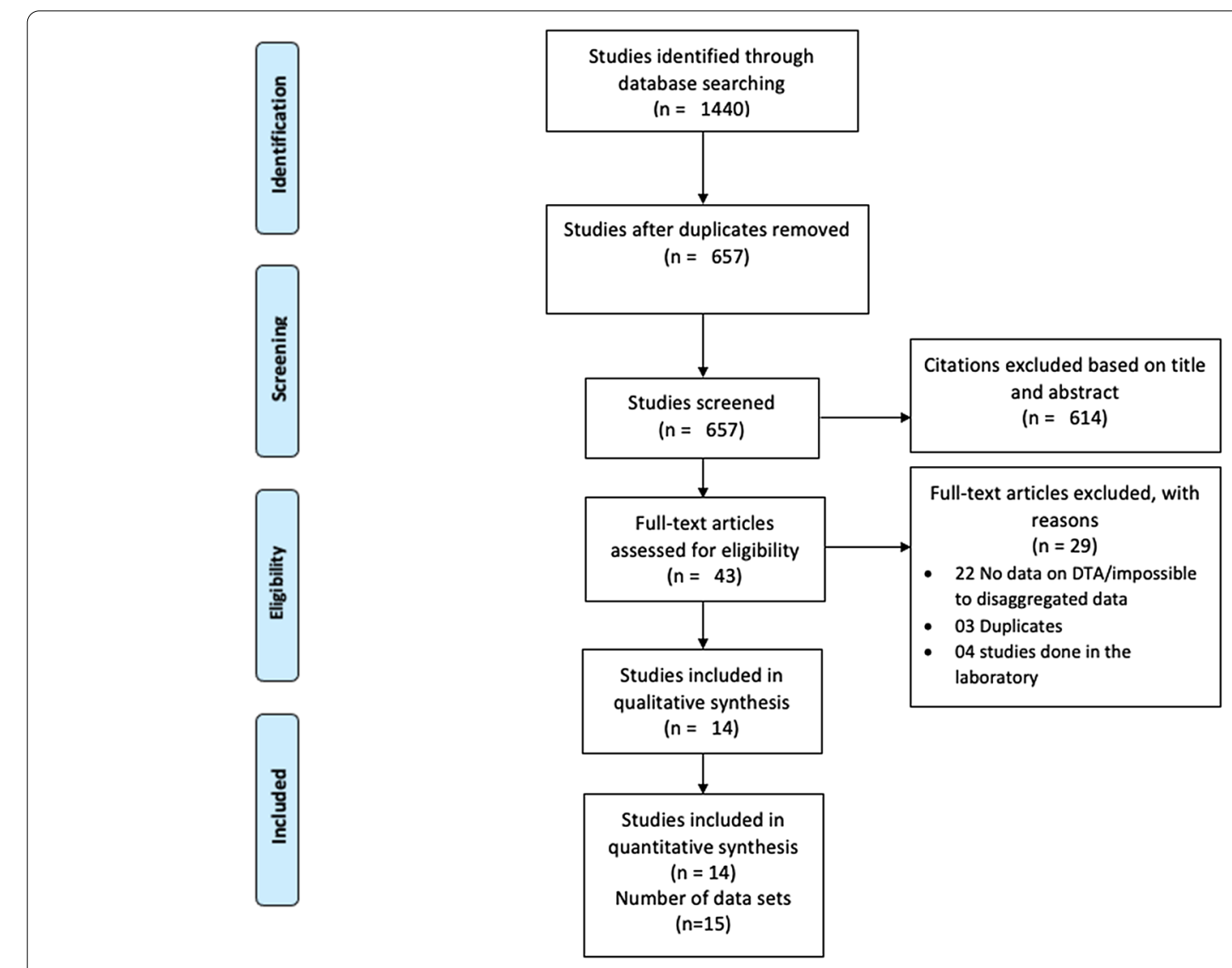

Fig. 1 The PRISMA flow diagram 


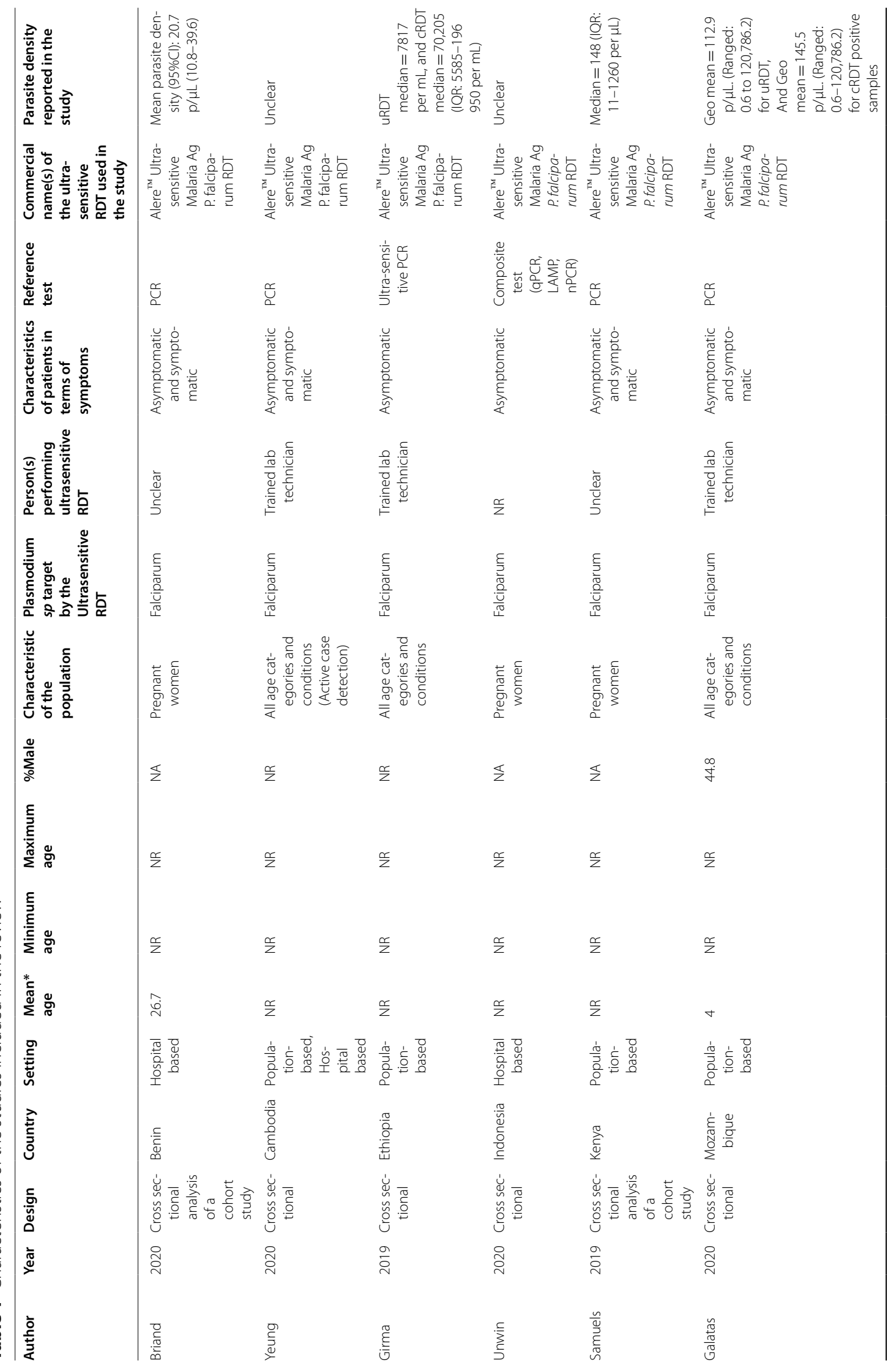




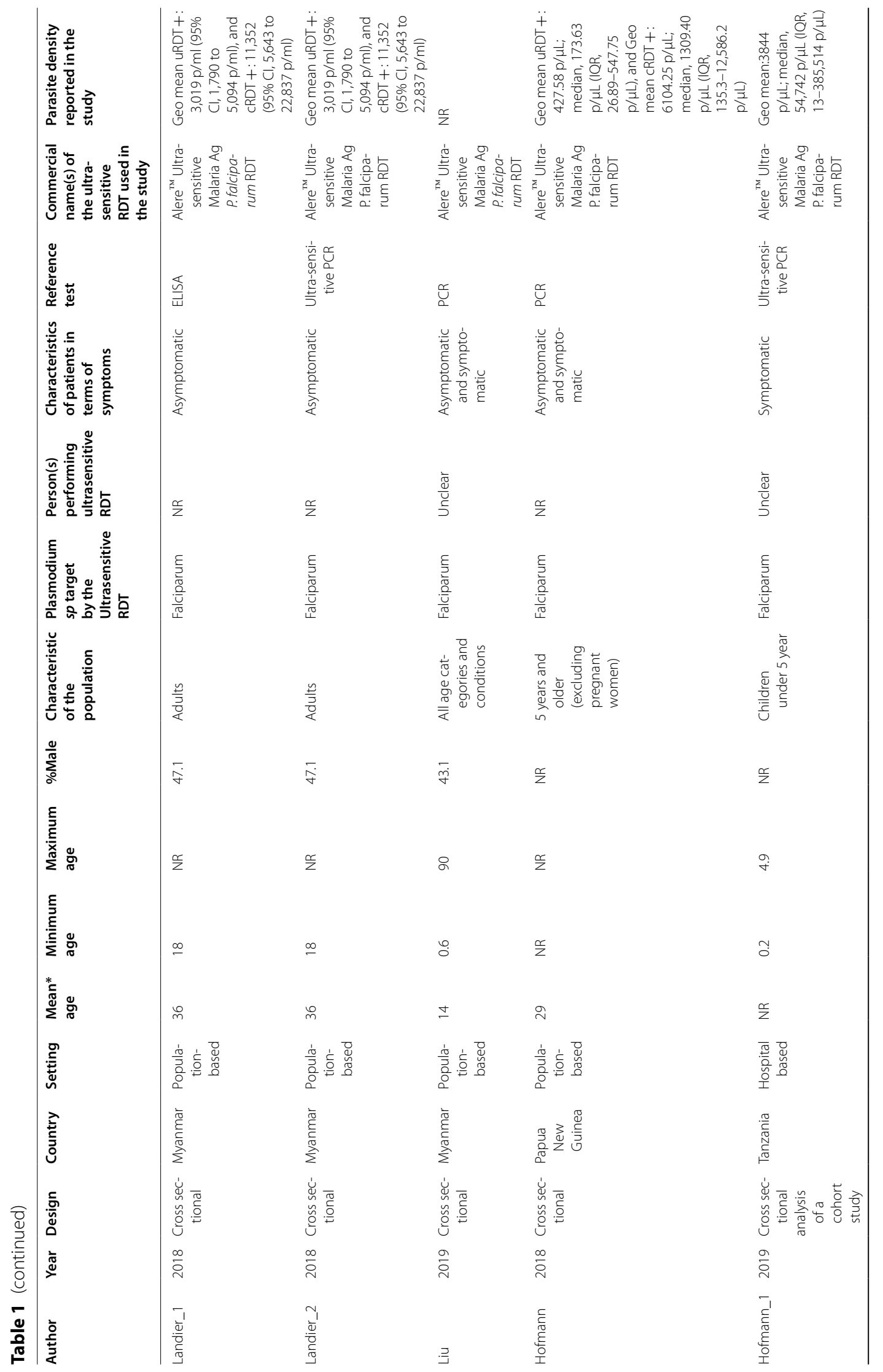




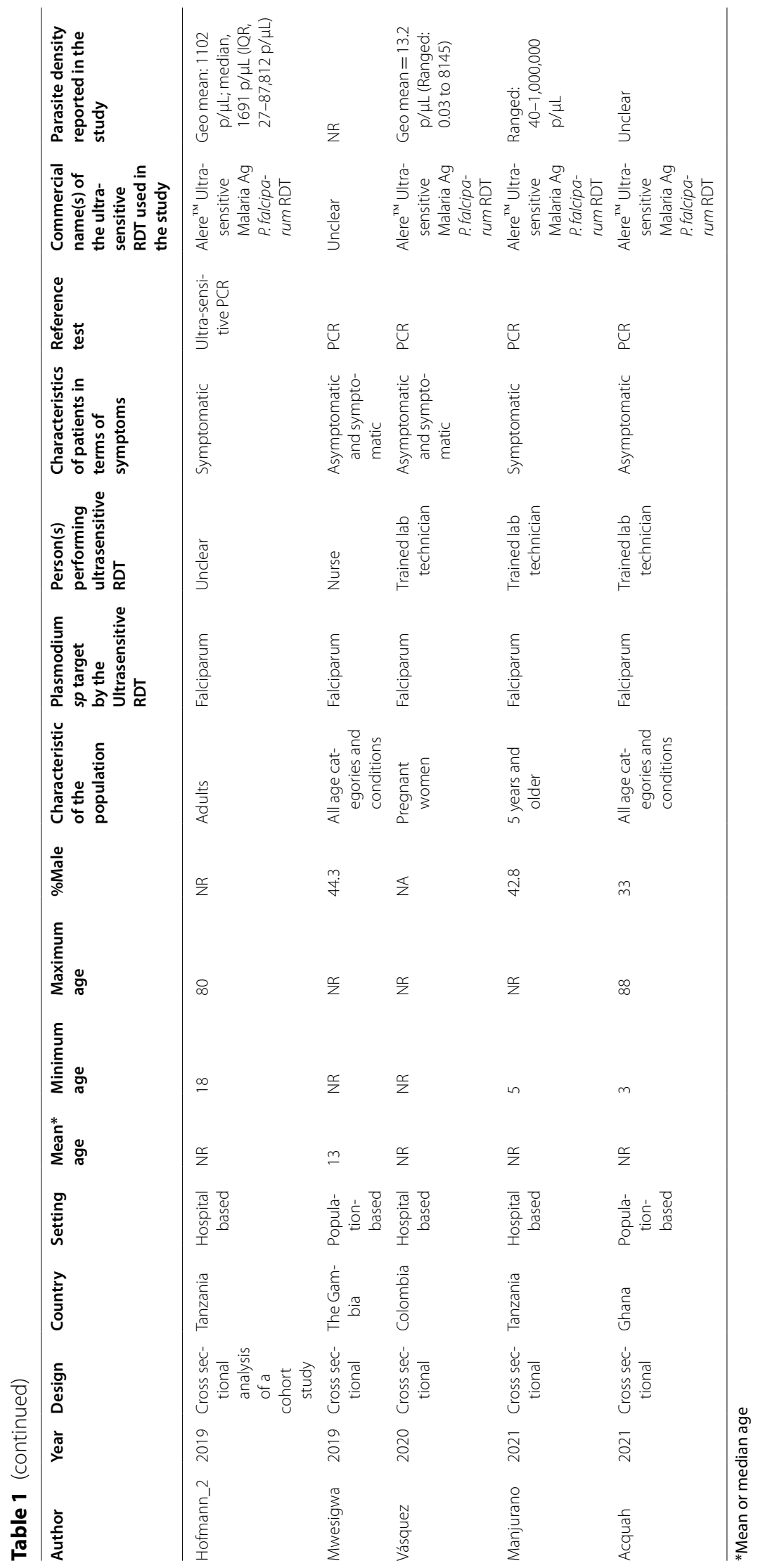




\section{Risk of Bias}

\section{Patient selection}

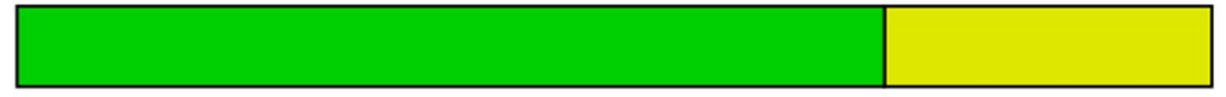

Index test

\section{Reference standard}

Flow \& timing

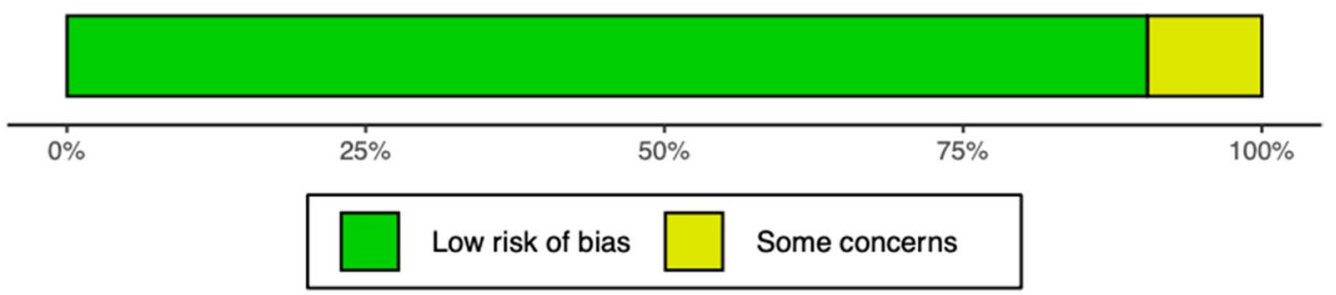

\section{Applicability concerns}

\section{Patient selection}

Index test

\section{Reference standard}

\section{Flow \& timing}

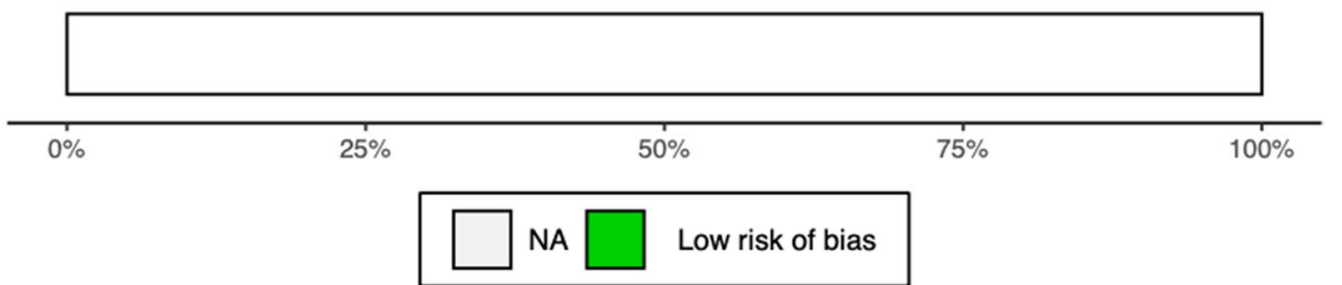

Fig. 2 Quality assessment of studies included in the meta-analysis

was $55.5 \%$ (95\% CI: $45.5 ; 65.0)$ while the figure was $42.9 \%$ (95\% CI: 31.5; 55.2) for the cRDT (Fig. 3). The difference in terms of sensitivity between the Alere ${ }^{\mathrm{TM}}$ Ultra-sensitive Malaria Ag P. falciparum RDT and cRDT varies according to the reference test used. When PCR was used as reference test, the sensitivity of Alere ${ }^{\mathrm{TM}}$ Ultra-sensitive Malaria Ag P. falciparum RDT was 60.4\% (95\% CI: 50.8; $69.2)$ while it was $49.4 \%$ (95\% CI: $38.2 ; 60.6)$ for the cRDT. When the ultra-sensitive PCR was used as reference test, sensitivity of uRDT was $60.3 \%$ (95\% CI: $42.2 ; 75.9)$ and of cRDT was $44.1 \%$ (95\% CI: 18.8; 72.8) (Table 2).

The sensitivity of Alere ${ }^{\mathrm{TM}}$ Ultra-sensitive Malaria Ag P. falciparum RDT and cRDT in pregnant women was 52.5\% (95\% CI: 31.3; 72.9) and 44.9\% (95\% CI:29.7; 61.2) respectively. Regardless of the reference test used, the sensitivity of Alere ${ }^{\mathrm{TM}}$ Ultra-sensitive Malaria Ag P. falciparum RDT in symptomatic patients was $72.1 \%$ (95\%CI: $67.4 ; 76.4$ ), while sensitivity of cRDT was $67.4 \%$ (95\%CI: 57.6; 75.9). In asymptomatic patients the sensitivity of Alere $^{\mathrm{TM}}$ Ultra-sensitive Malaria Ag $P$. falciparum RDT was $42.1 \%$ (95\%CI: 26.5 ; 59.5), and sensitivity of cRDT was $26.0 \%$ (95\%CI: $13.9 ; 43.4)$.

\section{Specificity}

The overall specificity of the Alere ${ }^{\mathrm{TM}}$ Ultra-sensitive Malaria Ag P. falciparum RDT was lower than the cRDT (statistically non-significant). The pooled specificity of Alere $^{\mathrm{TM}}$ Ultra-sensitive Malaria Ag P. falciparum RDT 


\section{A}

Study

Hofmann,2018 Landier_1,2018 Landier_2,2018 Girma,2019 Hofmann_1,2019
Hofmann_2,2019 Hofmann_2,20 Liu, 2019 Samuels, 2019 Briand,2020 Galatas,2020 Unwin, 2020 Yeung, 2020 Manjurano,2021 Manjurano,2021
Acquah,2021 Subgroup sensitivity Heterogeneity: $I^{2}=96.2 \%, p<0.000$

URDT

Hofmann,2018 Landier_1,2018 Landier_2,2018 Girma, 2019 Hofmann_1,2019 Hofmann_2,2019 Liu,2019 Samuels, 2019 Briand,2020 Galatas, 2020 Unwin, 2020 Yeung,2020 Vásquez,2020 Acquah,2021 Subgroup sensitivity Heterogeneity: $I^{2}=94.5 \%, p<0.000$
TP TP+FN Sensitivity, $\% \quad$ [95\% Cl]

$\begin{array}{lll}20 & 135 & 14.8[9.3 ; 21.9] \\ 30 & 198 & 15.2[10.5 ; 20.9]\end{array}$

$\begin{array}{lll}30 & 198 & 15.2[10.5 ; 20.9] \\ 31 & 183 & 16.9[11.8 ; 23.2]\end{array}$ $16.9[11.8 ; 23.2] \div$ $20.7[12.6 ; 31.1]$
$73.1[67.8 ; 78.0]$ $73.1[67.8 ; 78.0]$
$72.9[58.2 ; 84.7]$ $43.1[30.8 ; 56.0]$ $63.1[53.9 ; 71.7]$ $44.2[36.6 ; 51.9]$ $61.5[53.9 ; 68.6]$ $22.8[16.5 ; 30.1]$ $46.2[26.6 ; 66.6]$
$53.8[37.2 ; 69.9]$ $56.6[48.9 ; 64.0]$ $56.6[48.9 ; 64.0]$ $42.9[31.5 ; 55.2]$
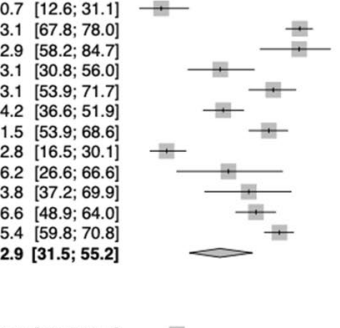

$26.7[19.4 ; 35.0]$
$34.8[28.2 ; 41.9]$
$36.6[29.6 ; 44.0]$ $36.6[29.6 ; 44.0]$
$50.0[38.7 ; 61.3]$ $74.4[69.2 ; 79.2]$ $77.1[62.7 ; 88.0]$ $55.4[42.5 ; 67.7]$ $69.7[60.7 ; 77.7]$ $60.5[52.7 ; 67.8]$ $68.2[60.8 ; 74.9]$
$19.6[13.7 ; 26.7]$ $19.6[13.7 ; 26.7]$
$53.8[33.4 ; 73.4]$ $64.1[47.2 ; 78.8]$ $64.1[47.2 ; 78.8$
$66.9[59.4 ; 73.8]$ $72.8[67.5 ; 77.8]$ $\mathbf{5 5 . 5}[\mathbf{4 5 . 5} ; \mathbf{6 5 . 0 ]}$

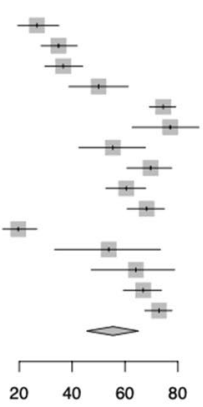

B

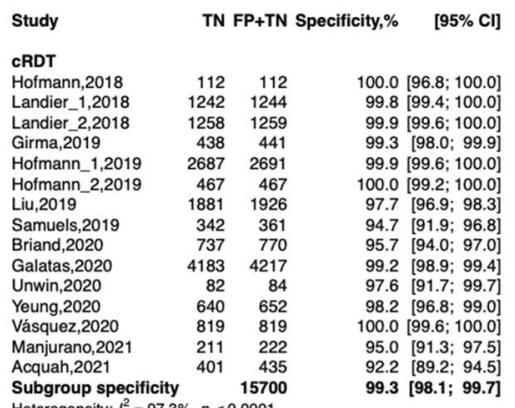

Specificity

Hofmann,2018 $\quad 112 \quad 112$

Landier_1,2018
Landier_2,2018

Girma, 2019

Hofmann_1,2019

Hofmann 2,2019

Liu, 2019

Samuels,2019

Briand,2020

Galatas, 2020

Unwin,2020

Yeung,2020

Vásquez,2020

Manjurano,202

Acquah,202

Subgroup specificity

$1238 \quad 1244$

$\begin{array}{rr}434 & 441\end{array}$

$\begin{array}{rr}4382 & 4691 \\ 467 & 467\end{array}$

$\begin{array}{rr}467 & 467\end{array}$

$\begin{array}{rr}1862 \quad 1926 \\ 341 & 361\end{array}$

$\begin{array}{ll}341 & 361 \\ 721 & 770\end{array}$

$\begin{array}{rr}4173 & 4217\end{array}$

$\begin{array}{rr}82 & 84 \\ 631 & 652\end{array}$

$\begin{array}{ll}631 & 652 \\ 813 & 814 \\ 207 & 222\end{array}$

207
389

$\begin{array}{rr}389 & 435 \\ 15695\end{array}$

Heterogeneity: $I^{2}=97.2 \%, p<0.0001$

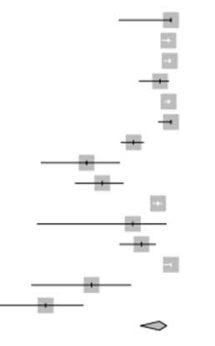

$00.0[96.8 ; 100.0]$

99.5 [99.0; 99.8$]$

$99.4[98.8 ; 99.7]$
$98.4[96.8 ; 99.4]$

98.4 [96.8; 99.4$]$

$100.0[99.4 ; 99.8]$

96.7 [95.8; 97.4]

$94.5[91.6 ; 96.6]$

93.6 [91.7; 95.3]

$99.0[98.6 ; 99.2]$

$97.6[91.7 ; 99.7]$

96.8 [95.1; 98.0]

93.2 [89.1; 96.2$]$

89.4 [86.1; 92.2]

$98.6[97.1 ; 99.4]$

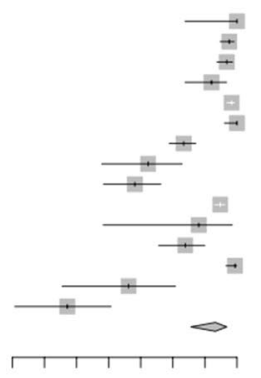

$\begin{array}{llllllll}86 & 88 & 90 & 92 & 94 & 96 & 98 & 100\end{array}$

Fig. 3 The forest plot of sensitivity and specificity

Table 2 Meta-analysis of diagnostic performance Alere ${ }^{\mathrm{TM}}$ Ultra-sensitive Malaria Ag P. falciparum RDT according to the reference test

\begin{tabular}{|c|c|c|c|c|c|c|c|c|c|}
\hline \multirow[t]{2}{*}{$\mathrm{N}$ data sets } & & \multicolumn{2}{|c|}{ Pooled sensitivity, \% [95\% Cl] } & \multicolumn{2}{|c|}{$\begin{array}{l}\text { Heterogeneity for } \\
\text { sensitivity }\left(I^{2}, \%\right)\end{array}$} & \multicolumn{2}{|c|}{ Pooled specificity, \% [95\% Cl] } & \multicolumn{2}{|c|}{$\begin{array}{l}\text { Heterogeneity } \\
\text { for } \\
\text { specificity }\left(I^{2} \text {, }\right. \\
\%)\end{array}$} \\
\hline & & uRDT & CRDT & uRDT & CRDT & uRDT & CRDT & uRDT & cRDT \\
\hline \multicolumn{10}{|c|}{ According to reference test } \\
\hline $\mathrm{PCR}$ & 9 & $60.4[50.8 ; 69.2]$ & $49.4[38.2 ; 60.6]$ & 89.4 & 92.6 & $97.5[94.1 ; 98.9]$ & $98.2[95.5 ; 99.3]$ & 97.6 & 97.1 \\
\hline Ultra-sensitive PCR & 4 & $60.3[42.2 ; 75.9]$ & $44.1[18.8 ; 72.8]$ & 93.6 & 97.1 & $99.5[98.9 ; 99.8]$ & $99.8[99.7 ; 99.9]$ & 71.9 & 0.0 \\
\hline
\end{tabular}

$P C R$ Polymerase chain reaction, $R D T$ Malaria rapid diagnostic test, $C I$ Confidence interval

was $98.6 \%$ (95\% CI: 97.1; 99.4), and of cRDT was $99.3 \%$ (95\% CI: 98.1; 99.7). When PCR was used as reference test the specificity of uRDT and cRDT was $97.5 \%(95 \%$ CI: 94.1 ; 98.9) and 98.2\% (95\% CI: 95.5; 99.3) respectively, and when the ultra-sensitive PCR was used as reference test the specificity was $99.5 \%(95 \% \mathrm{CI}: 98.9 ; 99.8)$ and 99.8\% (95\% CI: 99.7; 99.9), respectively (Table 2).

The specificity of Alere ${ }^{\mathrm{TM}}$ Ultra-sensitive Malaria Ag P. falciparum RDT and cRDT in pregnant women was 98.1\% (95\% CI: 91.5; 99.6) and 98.7\% (95\% CI: 90.9; 99.8), respectively. Regardless of the reference test used, the specificity of Alere $^{\mathrm{TM}}$ Ultra-sensitive Malaria Ag $P$. falciparum RDT in symptomatic patients was $99.5 \%$
(95\%CI: 92.6; 100.0), and specificity of cRDT was $99.7 \%$ (95\%CI:95.0; 100.0). In asymptomatic patients the specificity of Alere ${ }^{\mathrm{TM}}$ Ultra-sensitive Malaria Ag P. falciparum RDT was $98.4 \%$ (95\%CI: $95.4 ; 99.5)$, and specificity of cRDT was 99.3\% (95\%CI: 96.8; 99.9).

\section{Discussion}

This meta-analysis assessing the field performance of malaria uRDT (Alere ${ }^{\mathrm{TM}}$ Ultra-sensitive Malaria Ag P. falciparum RDT) highlights the higher sensitivity of the Alere $^{\mathrm{TM}}$ Ultra-sensitive Malaria Ag P. falciparum RDT compared to the cRDT when performed on the same field conditions and confirmed findings observed in 
the laboratory conditions [8]. Indeed, these results are promising for detection of malaria in patients with low parasitaemia, subclinical or asymptomatic infections and pregnant women. For the former because current cRDT available on the market and widely used in malaria endemic countries are not able to diagnose malaria in patients with a parasite density below $100 / \mu \mathrm{L}$ while uRDT can, this may explain the relatively higher sensitivity of uRDT [28]. For the latter because they are in most countries under intermittent preventive treatment (IPT), which can strongly influence the parasite density, and because of pathophysiology of malaria during pregnancy.

Indeed, in pregnant women, red blood cells parasite by Plasmodium bind to the chondroitin sulfate portion of syndecan- 1 of both intervillous space and the syncytiotrophoblast [5] leading to their sequestration into the placenta and explain a relative low blood parasite density $[29,30]$, and thus their capacity to escape to cRDT. Malaria in pregnancy is deleterious for both the mother [29] and the fetus [1]. For the mother, malaria can cause anaemia, severe disease, and death while for the fetus and newborn it contributes to stillbirth, preterm birth, and low birthweight $[1,5]$. The WHO estimated that 822,018 of cases of low birthweights in sub-Saharan Africa were related to exposure to malaria parasite during pregnancy in 2019. Given the tremendous burden of malaria in pregnant women, there is an urgent need of highly sensitive method that can help in timely efficient diagnosing of malaria in this vulnerable population. Interestingly the current meta-analysis found that uRDT performed better than CRDT in this specific population, which may allow to capture and treat additional cases that may have been missed by cRDT.

Even if the specificity of uRDT seems to be slightly lower than cRDT it is estimated to $98.6 \%$ (95\% CI: 97.1; 99.4) and is higher than 95\% regardless of the reference test used in blood. Importantly its specificity is not statistically significantly different from the one obtained for the cRDT in the current meta-analysis.

From a public health perspective, the findings of this study suggest that Alere ${ }^{\mathrm{TM}}$ Ultra-sensitive malaria $\mathrm{Ag} P$. falciparum RDT is more sensitive than cRDT and could help to capture additional low parasite density malaria cases that escape the current cRDT. These results call for the assessment of additional criteria, namely the stability at high temperature, cost and shelf life of uRDTs, before Alere $^{\mathrm{TM}}$ Ultra-sensitive malaria Ag $P$. falciparum RDT could be integrated into the already available malaria diagnostic arsenal. Furthermore, it is essential that a correlation be established in the field between the parasitaemia observed in patients and the positivity of uRDTs to confirm the results obtained in the laboratory conditions. Nevertheless, given that cRDTs typically do not consistently detect parasite densities lower than $100 \mathrm{p} / \mu \mathrm{L}$, the definition of an ultrasensitive malaria test needs to be clarified and the conditions to fulfil for a test to be considered ultrasensitive need to be consensually adopted to compliment the current WHO definition, which is based solely on the parasite density detection threshold (below 100 parasites/ $\mu \mathrm{l}$ ) [28].

The results of the current study must be interpreted considering some drawbacks. Most of the studies were conducted in WHO African region (sub-Saharan Africa), which is the region with the highest burden of malaria. This can limit the generalizability of the results to other malaria endemic regions of the world. Data does not allow for stratified analysis according to parasite density, which is one of the key elements in the performance of malaria diagnostic tests. None of the studies specified the storage conditions of uRDT and cRDT, and only seven reported clearly that the test was conducted by trained laboratory technician/nurse, this may have impacted on the quality of the results. Several brands of cRDT with different performances were used as comparators to the Alere $^{\mathrm{TM}}$ Ultra-sensitive Malaria Ag P. falciparum RDT in the current study. The diversity of these tests could be an additional source of heterogeneity in the results and may limit a direct comparison between Alere ${ }^{\mathrm{TM}}$ Ultra-sensitive Malaria Ag P. falciparum RDT and a specific brand of cRDT test. Nevertheless, this study is the first to assess by the mean of a meta-analysis, the performances of one of the most recent diagnostic tools of malaria diagnostic in the field conditions. Furthermore, recent guidelines were used for the assessment of quality of included studies and the reporting of the review.

\section{Conclusion}

Findings of the meta-analysis show that Alere ${ }^{\mathrm{TM}}$ Ultrasensitive Malaria Ag P. falciparum RDT compared to cRDT performed in the same field conditions has higher sensitivity but lower specificity although the difference is not statistically significant.

\section{Supplementary Information}

The online version contains supplementary material available at https://doi. org/10.1186/s12936-021-03783-2.

Additional file 1: Table S1. Search strategy for PubMed.

\section{Acknowledgements \\ None.}

Authors' contributions

CD, and SS selected the studies, extracted the relevant information, and synthesized the data. CD, FKS and SS did the literature search. CD performed analyses and wrote the first draft of the paper with inputs of FKS, and SS. SS conceived the original idea of the study with the inputs of CD and FKS. All 
authors critically revised successive drafts of the paper. All authors read and approved the final manuscript.

\section{Funding}

This study received no funding. However, as a PhD candidate, the corresponding author (CD) is receiving a scholarship from UCLouvain. Moreover, SS received a postdoctoral fellowship from the Fonds de recherche du Québec Santé (FRQS).

\section{Availability of data and materials}

All materials are available in the manuscript and additional file.

\section{Declarations}

\section{Ethics approval and consent to participate}

Not applicable.

\section{Consent for publication}

All authors approved the final version of the manuscript and agree for publication.

\section{Competing interests}

We declare no competing interests.

\section{Author details}

${ }^{1}$ Department of Surgery and Specialties, Faculty of Medicine and Biomedical Sciences, University of Yaoundé I, Yaoundé, Cameroon. ${ }^{2}$ Epidemiology and Biostatistics Unit, Institut de Recherche Expérimentale Et Clinique, Université Catholique de Louvain, Brussels, Belgium. ${ }^{3}$ Centre for Research in Epidemiology, Biostatistics and Clinical Research, School of Public Health, Université Libre de Bruxelles (ULB), Route de Lennik, 808, 1070 Bruxelles, Brussels, Belgium. ${ }^{4}$ Centre for Research On Planning and Development (CRAD), Laval University, Quebec G1V 0A6, Canada. ${ }^{5}$ Evaluation Platform On Obesity Prevention, Quebec Heart and Lung Institute, Quebec G1V 4G5, Canada.

Received: 4 January 2021 Accepted: 25 May 2021

Published online: 03 June 2021

\section{References}

1. WHO. World Malaria Report 2020. Geneva, World Health Organization, 2020. https://www.who.int/teams/global-malaria-programme/reports/worldmalaria-report-2020. Accessed 23 Dec 2020.

2. Danwang C, Bigna JJ, Nzalie RNT, Robert A. Epidemiology of clinical congenital and neonatal malaria in endemic settings: a systematic review and meta-analysis. Malar J. 2020;19:312.

3. Rogerson SJ, Desai M, Mayor A, Sicuri E, Taylor SM, van Eijk AM. Burden, pathology, and costs of malaria in pregnancy: new developments for an old problem. Lancet Infect Dis. 2018;18:e107-18.

4. WHO. T3: Test. Treat. Track. Scaling up diagnostic testing, treatment and surveillance for malaria. Geneva: World Health Organization; 2012. https:// www.who.int/malaria/publications/atoz/t3_brochure/en/. Accessed 23 Dec 2020

5. Sharma L, Shukla G. Placental malaria: a new insight into the pathophysiology. Front Med (Lausanne). 2017;4:117.

6. WHO. Policy brief on malaria diagnostics in low-transmission settings. Geneva:World Health Organization; 2014. http://www.who.int/malaria/ publications/atoz/policy-brief-diagnosis-low-transmission-settings/en/. Accessed 23 Dec 2020

7. Zainabadi K, Adams M, Han ZY, Lwin HW, Han KT, Ouattara A, et al. A novel method for extracting nucleic acids from dried blood spots for ultrasensitive detection of low-density Plasmodium falciparum and Plasmodium vivax infections. Malar J. 2017;16:377.

8. Das S, Jang IK, Barney B, Peck R, Rek JC, Arinaitwe E, et al. Performance of a high-sensitivity rapid diagnostic test for Plasmodium falciparum malaria in asymptomatic individuals from Uganda and Myanmar and naive human challenge infections. Am J Trop Med Hyg. 2017;97:1540-50.

9. Cochrane Handbook for Systematic Reviews of Diagnostic Test Accuracy. 2010. /resource/cochrane-handbook-systematic-reviews-diagnostic-testaccuracy. Accessed 22 Dec 2020.

10. Mclnnes MDF, Moher D, Thombs BD, McGrath TA, Bossuyt PM, and the PRISMA-DTA Group. Preferred reporting items for a systematic review and meta-analysis of diagnostic test accuracy studies: the PRISMA-DTA statement. JAMA. 2018:319:388-96.

11. Whiting PF. QUADAS-2: a revised tool for the quality assessment of diagnostic accuracy studies. Ann Intern Med. 2011;155:529-36.

12. Doebler P. mada: meta-analysis of diagnostic accuracy. 2020. https:// CRAN.R-project.org/package=mada. Accessed 22 Dec 2020.

13. meta-package function. R Documentation https://www.rdocumentation. org/packages/meta/versions/4.15-1/topics/meta-package. Accessed 23 Dec 2020

14. Liu Z, Soe TN, Zhao Y, Than A, Cho C, Aung PL, et al. Geographical heterogeneity in prevalence of subclinical malaria infections at sentinel endemic sites of Myanmar. Parasit Vectors. 2019;12:83.

15. Landier J, Haohankhunnatham W, Das S, Konghahong K, Christensen P, Raksuansak J, et al. Operational performance of a Plasmodium falciparum ultrasensitive rapid diagnostic test for detection of asymptomatic infections in Eastern Myanmar. J Clin Microbiol. 2018;56:e00565-e618.

16. Hofmann NE, Antunes Moniz C, Holzschuh A, Keitel K, Boillat-Blanco N, Kagoro F, et al. Diagnostic performance of conventional and ultrasensitive rapid diagnostic tests for malaria in febrile outpatients in Tanzania. J Infect Dis. 2019;219:1490-8.

17. Manjurano A, Omolo JJ, Lyimo E, Miyaye D, Kishamawe C, Matemba LE, et al. Performance evaluation of the highly sensitive histidine-rich protein 2 rapid test for Plasmodium falciparum malaria in North-West Tanzania. Malar J. 2021:20:58.

18. Briand V, Cottrell G, Tuike Ndam N, Martiáñez-Vendrell X, Vianou B, Mama A, et al. Prevalence and clinical impact of malaria infections detected with a highly sensitive HRP2 rapid diagnostic test in Beninese pregnant women. Malar J. 2020;19:188.

19. Yeung S, McGregor D, James N, Kheang ST, Kim S, Khim N, et al. Performance of ultrasensitive rapid diagnostic tests for detecting asymptomatic Plasmodium falciparum. Am J Trop Med Hyg. 2020;102:307-9.

20. Girma S, Cheaveau J, Mohon AN, Marasinghe D, Legese R, Balasingam N, et al. Prevalence and epidemiological characteristics of asymptomatic malaria based on ultrasensitive diagnostics: a cross-sectional study. Clin Infect Dis. 2019:69:1003-10.

21. Unwin VT, Ahmed R, Noviyanti R, Puspitasari AM, Utami RAS, Trianty L, et al. Use of a highly-sensitive rapid diagnostic test to screen for malaria in pregnancy in Indonesia. Malar J. 2020;19:28

22. Samuels AM, Towett O, Seda B, Onoka K, Otieno K, Chebore W, et al. Diagnostic performance of ultra-sensitive rapid diagnostic tests for malaria in pregnant women attending antenatal clinics in Western Kenya. Am J Trop Med Hyg. 2019;101:276.

23. Galatas B, Mayor A, Gupta H, Balanza N, Jang IK, Nhamussua L, et al. Field performance of ultrasensitive and conventional malaria rapid diagnostic tests in southern Mozambique. Malar J. 2020;19:451.

24. Hofmann NE, Gruenberg M, Nate E, Ura A, Rodriguez-Rodriguez D, Salib $\mathrm{M}$, et al. Assessment of ultra-sensitive malaria diagnosis versus standard molecular diagnostics for malaria elimination: an in-depth molecular community cross-sectional study. Lancet Infect Dis. 2018;18:1108-16.

25. Acquah FK, Donu D, Obboh EK, Bredu D, Mawuli B, Amponsah JA, et al. Diagnostic performance of an ultrasensitive HRP2-based malaria rapid diagnostic test kit used in surveys of afebrile people living in Southern Ghana. Malar J. 2021:20:125

26. Vásquez AM, Vélez G, Medina A, Serra-Casas E, Campillo A, Gonzalez IJ, et al. Evaluation of highly sensitive diagnostic tools for the detection of $P$. falciparum in pregnant women attending antenatal care visits in Colombia. BMC Pregnancy Childbirth. 2020;20:440.

27. Mwesigwa J, Slater H, Bradley J, Saidy B, Ceesay F, Whittaker C, et al. Field performance of the malaria highly sensitive rapid diagnostic test in a setting of varying malaria transmission. Malar J. 2019;18:288. 
28. WHO MPACM. Meeting report of the WHO Evidence Review Group on Low-Density Malaria Infections. Geneva:World Health Organization; 2017. https://www.who.int/malaria/mpac/mpac-oct2017-erg-malaria-low-dens ty-infections-session2.pdf

29. Cohee LM, Kalilani-Phiri L, Mawindo P, Joshi S, Adams M, Kenefic L, et al. Parasite dynamics in the peripheral blood and the placenta during pregnancy-associated malaria infection. Malar J. 2016;15:483.
30. Clark RL. Genesis of placental sequestration in malaria and possible targets for drugs for placental malaria. Birth Defects Res. 2019;111:569-83.

\section{Publisher's Note}

Springer Nature remains neutral with regard to jurisdictional claims in published maps and institutional affiliations.
Ready to submit your research? Choose BMC and benefit from:

- fast, convenient online submission

- thorough peer review by experienced researchers in your field

- rapid publication on acceptance

- support for research data, including large and complex data types

- gold Open Access which fosters wider collaboration and increased citations

- maximum visibility for your research: over $100 \mathrm{M}$ website views per year

At BMC, research is always in progress.

Learn more biomedcentral.com/submissions 